\title{
Grasp mode and compliance control of an under-actuated origami gripper using adjustable stiffness joints
}

\author{
Amir Firouzeh, Student member, IEEE, and Jamie Paik, Member, IEEE,
}

\begin{abstract}
Every robotic gripper requires an equilibrated solution towards the grasp adaptability, precision, and load bearing capacity. A versatile soft robotic gripper requires adjustable grasp mode, for objects with different sizes and shapes, and adjustable compliance, for switching between soft mode; for small loads and delicate objects; and stiff mode; for larger loads and heavier objects. In this paper, we present the design of a tendon-driven robotic origami, robogami, gripper that provides self-adaptability and inherent softness through its redundant and under-actuated degrees of freedom (DoF). Robogami is a planar and foldable robotic platform that is scalable and customizable thanks to its unique layer-by-layer manufacturing process. The nominally 2D fabrication process allows embedding different functional layers with a high fidelity. In particular, a polymer layer with adjustable stiffness enables the independent control of the stiffness for each joint. Using this feature, we can control the input energy distribution between different joints and hence the motion of the robogami. Here, we model the behavior of a single finger; and demonstrate the compliance control of the endeffector along different directions in simulations and experiments. We also validate the gripper's task versatility in soft and stiff modes by assigning model-based joints stiffness for performing different grasp modes.
\end{abstract}

Index Terms-Robotic origami, layer-by-layer manufacturing, tendon driven joints, adjustable stiffness joints, under-actuated gripper, shape memory polymer, compliance control.

\section{INTRODUCTION}

Robotics Origamis, robogamis, are a family of foldable robots that achieve conformity by their multiple degrees of freedom (DoFs) which are driven by soft actuation methods [1]-[4]. The DoFs in robogamis are defined by the folding pattern of a polymer sheet which serves as the hinge layer [1]. The folding pattern is determined by the rigid layers that embed the polymer layer. Layers with different functions are integrated in robogamis thanks to their layer-by-layer manufacturing [2]. The precise fabrication methods for machining quasi-2D layers allows us to design robots with many DoFs in confined space. In this research we focus on tendon-driven robogamis. In these robots, a single source of actuation drives multiple joints. The redundant DoFs and the under-actuation make these robots inherently soft and safe for interaction with sensitive environment. Our goal is to use the tendon-driven robogami platform as a versatile soft gripper.

Soft grippers and hands are among the applications that benefit from the advantages of soft robots such as adaptability, safe interaction, low cost, customizabitiy, and simplicity of

A.Firouzeh and J. Paik are with the Reconfigurable Robotics Lab, EPFL, Lausanne, Switzerland e-mail: Jamie.paik@epfl.ch. actuation and control [5]-[8]. The compliance of the soft fingers in these robots allows them to conform to the shape of the objects for applying contact forces at the proper points and directions without the need for complex sensors, actuators, and controllers. However, there is a compromise to make between the compliance and the force output [9]. Soft actuation methods with adjustable stiffness solve this problem by enabling the assignment of different compliance to the robotic hand fingers depending on the task. Different physical principles for controlling the stiffness of the robot segments and joints have been proposed in the literature ranging from the mechanical methods of stiffness control [10]-[17] to methods that rely on the material properties [4], [6], [18]-[28]. The method of stiffness control in robogamis should be scalable and compatible with its layer-by-layer manufacturing. This rules out most of the mechanical methods of stiffness control and some of the methods that are based on the material properties. Among the remaining options, using polymer properties around the glass transition temperature is the most effective choice given the significant change in the stiffness, low price, ease of processing, and compatibility with the quasi-2D fabrication methods. Shape memory polymers (SMP) offer a significant stiffness modulation and a high shape recovery rate which makes them a desirable choice among other thermoplastics. In this research, SMP pellets (MM 5520 from SMP Technologies Inc.) are formed into layers and are embedded in the structure for controlling the stiffness. To improve the shape recovery, the SMP layer is encapsulated in silicone rubber as suggested in [21], [29]. For the material activation, stretchable metallic heaters are embedded in the SMP layer for controlling its temperature and hence the stiffness of the joints. The fabrication process of the stretchable metal mesh structures and their integration process have been presented in detail in [18]. The adjustable stiffness layer (ASL) which controls the joint stiffness in the robogamis consists of a stretchable heater, SMP, and silicone rubber. The temperature and the stiffness of the ASL are tuned by adjusting the input power to the stretchable heater [29]. We previously confirmed the durability of the ASL layer in few hundred cycles of loading [21]. We also characterized the stiffness of the ASL at different temperatures and verified significant stiffness variability, from $110 \mathrm{~N} / \mathrm{mm}$ at room temperature to $2.6 \mathrm{~N} / \mathrm{mm}$ at $110^{\circ} \mathrm{C},[29]$. This variation in the stiffness can be effectively used to control the reconfiguration and compliance of robogamis.

In this paper, we present a gripper based on the tendon- 
driven robogamis with adjustable stiffness joints. The operation mode of the gripper is assigned by adjusting the temperature of the ASLs in each joint which is rather slow, around 15 seconds, mainly due to the limited cooling rate. The actuation of the gripper in each mode, however, can be much faster and the gripper can be effectively used at few Hz. The variable mode of operation in this versatile gripper allows it to perform diverse tasks that require varying levels of compliance and different trajectories for each finger. The main contributions of the paper are:

- A novel concept of soft actuation with adjustable compliance in tendon-driven robogamis.

- The model of the robogami finger actuation and study of its motion at different stiffness settings for the joints and external loads.

- A prototype of a soft robogami gripper with adjustable compliance and demonstration of the versatility of the grasp modes.

The layer-by-layer manufacturing of the robogamis allows us to easily scale down the size of the gripper presented here or change its design for additional DoFs. The adjustable stiffness of the robogami joints enables us to set a desired initial configuration and set proper stiffness for active joints for performing tasks that require different grasping motions and compliance settings.

In Section II, the design of the robogami joints and their manufacturing process is presented. In Section III, we model the relation between the joints stiffness, the contact forces, and the tendon tension in an under-actuated robogami. We use the model to simulate the behavior of a single finger under load and demonstrate the compliance control. We also validate the simulation results with experiments in this Section. In Section IV, the gripper design is presented and different grasp modes corresponding to different compliance settings for the fingers are demonstrated. Finally in Section V, we conclude the findings and suggest the future steps.

\section{DESIGN AND MANUFACTURING OF THE TENDON-DRIVEN ROBOGAMI JOINTS WITH ADJUSTABLE STIFFNESS}

Soft and adaptable robot interaction with the environment requires multiple controllable DoFs. The layer-by-layer manufacturing of robogamis along with the precise methods for fabricating each individual layer allows us to design and fabricate robots with multiple DoFs in confined space. Moreover, this manufacturing method enables us to integrate layers with different functions in the structure. The DoFs in robogamis are defined by the folding pattern of a polyimide sheet. The patterns of the rigid layers, mostly micro-machine glass or carbon fiber layers, that cover the polyimide layer determines the arrangement of DoFs. Fig. 1a presents different layers that construct a robogami structure with two joints. The quasi2D layers are micro-machined using a UV laser (for details refer to [3]). These layers are bonded together using thermoset adhesive, Pyralux LF0100, to form the robogami as presented in Fig. 1b. Next, the outer structure which is used for aligning the layers during the bonding process is cut to release the

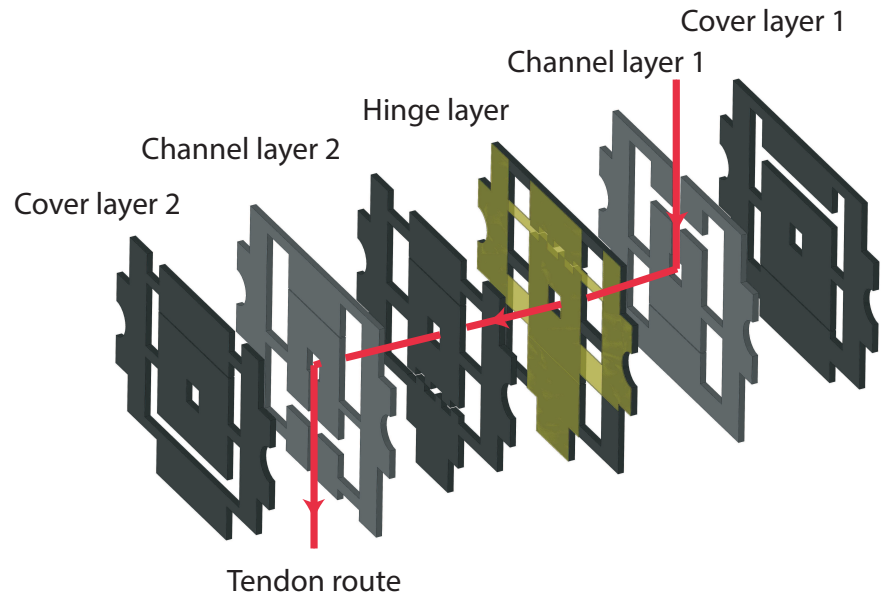

(a)

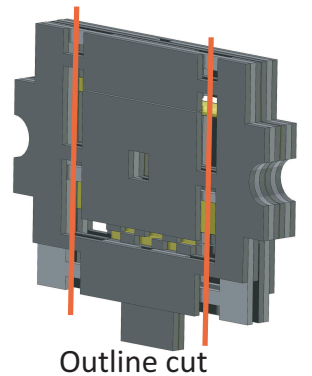

(b)

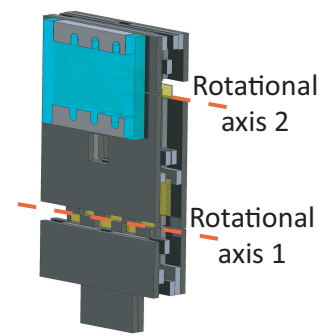

(c)
Fig. 1: The layer-by-layer manufacturing process of two robogami joints with opposite directions of actuation. Different layers are fabricated using accurate quasi-2D machining processes. The red line in this figure shows the path of the the tendon and highlights how it switches sides from the first to the second joint (a). The layers are aligned using the external alignment structure and are bonded together in a heat press using thermoset adhesive layers (b). The outer alignment structure is cut to release the joints and the ASLs are glued at the proper place (c).

joints and the ASLs are glued on top of the joints as presented in Fig. 1c. The castellated pattern of the glass-fiber layers define the joint axis and also the joints range of motion [29]. The design of the gripper presented in this paper is based on under-actuated robogamis. In these robots, a tendon that runs throughout the joints, actuates all DoF. In the example of Fig. 1 , the tendon route passes through both joints. The tendon switches side between the first and the second joint. So the joints of the robogami presented in Fig. 1c are actuated in opposite directions. Fig. 2 presents an schematic of the design and illustrates the tendon path between the two joints.

We control the ratio of the power transmission to different joints in under-actuated robogamis through adjusting the joints stiffness. The adjustable stiffness layer (ASL) which is illustrated in Fig. 1c consists of a customized stretchable heater, SMP, and silicone rubber. The stiffness of the ASL decreases more than 40 times as its temperature increases from the room temperature to $110{ }^{\circ} \mathrm{C}$ [29]. Using this variation in the stiffness, we can control the mechanical properties of the joints and the overall compliance of the structure.

The ASLs in both joints of the robogami presented in Fig. 2 are placed in the tension side of the neutral plane. If the ASL were placed on the compression side, it would buckle 


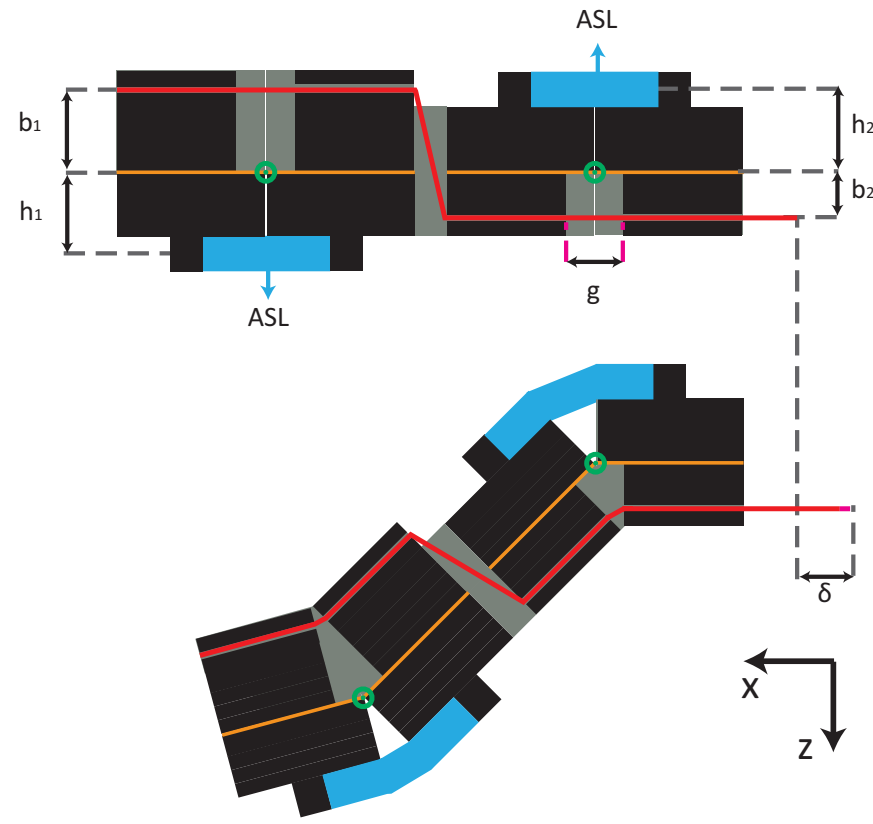

Fig. 2: The schematic of two robogami joints which are actuated in the opposite directions. The tendon (marked in red) switches sides after the first joint and actuates the second joint in the opposite direction. The ASLs for the first and second joints are placed at the same distance from the neutral plane but on the opposite sides in order to be on the tension side of each joint. The distance of the tendon from the joint axis can be adjusted in the design based on the required actuation moment for each joint.

under load and would not contribute considerably to the joint stiffness. Given the opposite direction of motion of the two joints, the ASLs are placed at the same distance but on the opposite sides. The tendon, however, is routed at different distance for the first joint and the second joint which allow us to use these joints more effectively as will be discussed in Section III. The joint design in Fig. 1 and 2 is similar to the design of the joints in the gripper and the design parameters and their values are presented in Table I. The negative values for $h_{1}$ and $b_{1}$ signify the opposing direction of actuation for the first joint.

The moment in the robogami joints is transferred through the tension and compression of the polyimide and the ASLs in the joints. The main failure modes for these joints is the over strain in the hinge layer and the buckling of the ASL. Note that given the small length of the polyimide layer between the tips of the castellated pattern on the two sides, buckling is not a failure mode for this layer. The hinges layer is a $125 \mu \mathrm{m}$ Polyimide sheet. Considering the ultimate strength of the material (169 $\mathrm{kpa}$ ) this layer can withstand $21.3 \mathrm{~N} / \mathrm{mm}$ for its unit width. So the maximum moment that the hinge layer can withhold is 970 N.mm. The second mode of failure is the buckling of the SMP layer. In its stiff mode, the thin SMP layer is the main load bearing element in the ASL. As a conservative assumption, we neglect the effect of the silicone layer in the following analysis and calculate the critical buckling load for the SMP layer:

$$
F_{\text {bcuckling }}=\frac{n \pi^{2} E I}{L^{2}}
$$

The thickness of the SMP layer is $500+50 \mu \mathrm{m}$ and its
TABLE I: The values of the design parameters.

\begin{tabular}{|c|c|c|}
\hline Parameters & Value $(\mathrm{mm})$ & Description \\
\hline$l_{p n}$ & 8.2 & the length of $2^{\text {nd }}-6^{\text {th }}$ and $8^{\text {th }}$ links \\
\hline$l_{p l}$ & 11.4 & the length of $1^{\text {st }}$ and $7^{\text {th }}$ links \\
\hline$g$ & 4 & Gap size in the middle part \\
\hline$w_{A S L}$ & 14 & ASL width in the active part \\
\hline$l_{A S L}$ & 4 & ASL length in the active part \\
\hline$t_{A S L}$ & 2 & ASL thickness \\
\hline$t_{S M P}$ & 0.5 & ASL thickness \\
\hline$h_{1}$ & 3.25 & ASL distance from the axis $2-7$ \\
\hline$h_{2}$ & -3.25 & ASL distance from the axis $2-7$ \\
\hline$b_{1}$ & 3.5 & Tendon distance moment arm $2-7$ \\
\hline$b_{2}$ & -2.5 & Tendon distance moment arm 1 and 8 \\
\hline
\end{tabular}

modulus of elasticity in the glassy state is $2.1 \mathrm{GPa}$. (1) predicts the critical load to be $42.8 \mathrm{~N} / \mathrm{mm}$ for the unit width of the SMP layer which is twice the maximum allowed force for the polyimide sheet. So the load bearing capacity of the joints is determined by the strength of the polyimide layer, $970 \mathrm{~N} . \mathrm{mm}$ for the design values of Table I. A sequence of the joints presented in this Section is used in the fingers of the gripper. To determine the design, we used the model to study the motion of under-actuated finger with different DoFs. In the next Section, we present the sequence of the joints we used for the gripper fingers and study the behavior of the finger with different compliance settings in simulation and experiment.

\section{TENDON-DRIVEN ROBOGAMI FINGER WITH ADJUSTABLE STIFFNESS JOINTS}

Any sequences of the joints presented in the previous Section can be designed and fabricated to make a robogami with desired workspace and reconfiguration. The main goal in this research is to demonstrate soft actuation and compliance control of robogami fingers and to implement them in an under-actuated gripper. Based on this, we designed a sequence of joints, presented in Fig. 3, that allows us to significantly change the compliance and behavior of the robogami fingers. We confined the design to the motion in plane. So all the joints have parallel axis. This simplifies the theoretical and experimental analysis.

The redundant DoFs of the finger increases its conformity and shape adaptability which is a necessity for a soft gripper. The stiffness control allows us to switch the mode of operation to stiffer and more precise motions by locking the joints in position or activating them at lower compliance. The first and the last joints in this design are actuated in the opposite direction compared to the rest of the joints. The range of motion for these two joint is set to $\left[-45^{\circ} 0^{\circ}\right]$ and for the rest

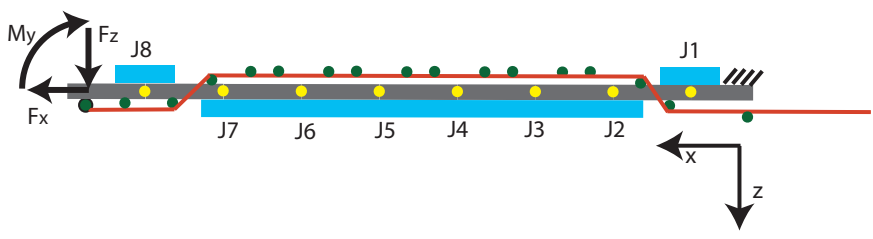

Fig. 3: The schematic of the robogami finger. The tendon (marked red) changes side about the hinge layer first between the $1^{\text {st }}$ and the $2^{\text {nd }}$ joints and next between the $7^{\text {th }}$ and the $8^{\text {th }}$. The ASL is placed on the opposite side of the tendon in all joints to be on the tension side. 
of the joints, joints $2-6$, the range of motion is set to $\left[0^{\circ} 45^{\circ}\right]$. In general the activation of more joints makes the structure more compliant. The activation of the first or the last joint, however, has more significant influence on the compliance which originates from their opposite direction of actuation. This allows us to switch between different modes to achieve either higher forces or more adaptability and compliance of actuation. We assigned a smaller tendon distance from the joint axis for the first and the eighth joints. This limits the actuation moment for these two joints which allow us to use them more effectively to control the stiffness of the end-effector. The overall length of the finger is $85 \mathrm{~mm}$ and the distance of the first joint from the tip is $72 \mathrm{~mm}$. So based on the ultimate strength of the joints calculated in the previous Section, we expect the finger to withstand maximum load of $13 \mathrm{~N}$ in z direction. We tested the finger upto $4.4 \mathrm{~N}$.

The reconfiguration and the mode of operation of an underactuated robogami is determined by the stiffness assigned to its joints. Here, we present a model which enables us to simulate the motion and the stable configuration of the robogami fingers based on the joints stiffness and contact forces. Based on the desired tasks for the gripper, we used this model to determine the proper joint sequence in the gripper and to find the proper joints stiffness for performing different tasks. In what follows, we present three examples of the simulations performed to study the compliance of the end-effector of the robogami finger in different directions. These examples correspond to different grasp modes that are presented in the next section.

To find the stable configuration of the finger, we equate the input work with the sum of the required work for changing the configuration of the robogami and the work for moving the end-effector against the contact forces:

$$
\boldsymbol{F}_{\boldsymbol{t}}^{T} \dot{\boldsymbol{X}}=W_{\text {contact }}+W_{A S L}
$$

In (2), the left side represents the input work. The elements of $\boldsymbol{F}_{\boldsymbol{t}}$ are the tension in the tendon at each joint. At this stage, we neglect the tendon friction and consider constant tendon tension for all joints. The elements of $\dot{\boldsymbol{X}}$ are the rate of the tendon displacement caused by the motion of each joint. The first term in the right side of this equation, $W_{\text {contact }}$, corresponds to the work done by the contact forces. The second term on the right side, $W_{A S L}$, correspond to the work done to reconfigure the ASLs. $W_{\text {contact }}$ is calculated as:

$$
W_{\text {contact }}=\left[F_{x}, F_{z}, M_{y}\right] \mathbf{J} \dot{\boldsymbol{\theta}}
$$

In which $F_{x}$ and $F_{z}$ are the contact forces in $x$ and $z$ directions and $M_{y}$ is the moment applied to the last link. The Jacobian matrix, $\mathbf{J}$, relates the motion of the end-effector to the rotation of the joints, $\dot{\boldsymbol{\theta}}$, and is calculated as:

$$
\mathbf{J}=\left[\begin{array}{ccc}
\sum_{j=1}^{n} l_{j} \sin \left(\sum_{i=1}^{j} \theta i\right) & \ldots & l_{n} \sin \left(\sum_{i=1}^{n} \theta_{i}\right) \\
\sum_{j=1}^{n} l_{j} \cos \left(\sum_{i=1}^{j} \theta i\right) & \ldots & l_{n} \cos \left(\sum_{i=1}^{n} \theta_{i}\right) \\
1 & \ldots & 1
\end{array}\right]
$$

The rate of the tendon displacement, $\dot{\mathbf{X}}$ in (2), has the following relation with the angular velocities of the joints:

$$
\dot{\mathbf{X}}=\boldsymbol{T} \dot{\boldsymbol{\theta}}=\left[\begin{array}{cccc}
\frac{\partial X_{1}}{\partial \theta_{1}} & \ldots & 0 & 0 \\
0 & \frac{\partial X_{2}}{\partial \theta_{2}} & \ldots & 0 \\
: & : & : & : \\
0 & 0 & \ldots & \frac{\partial X_{8}}{\partial \theta_{8}}
\end{array}\right] \dot{\boldsymbol{\theta}}
$$

$\frac{\partial X_{i}}{\partial \theta_{i}}$, in (5), is the transmission ratio between the input and the $i^{t h}$ joint. For the correlation between the rate of the joint angle change and the rate of tendon displacement for each joint, we have:

$$
d X_{i}=-\frac{X_{i_{x}} b+X_{i_{y}} \frac{g}{2}}{\sqrt{{X_{i_{x}}{ }^{2}+X_{i_{y}}{ }^{2}}^{2}}} d \theta_{i}
$$

in which $X_{i_{x}}$ and $X_{i_{y}}$ are solved as:

$$
\begin{aligned}
X_{i_{x}} & =\frac{g}{2}\left(1+\cos \theta_{i}\right)-b \sin \theta_{i} \\
X_{i_{y}} & =\frac{g}{2}\left(\sin \theta_{i}\right)+b \cos \theta_{i}-b
\end{aligned}
$$

Note that (6)-(8) are valid for joints in both directions as long as the sign of the tendon distance from the axis of rotation is considered correctly. The required work for changing the configuration of the finger, $W_{A S L}$, is calculated as:

$$
W_{A S L}=\boldsymbol{F}_{\boldsymbol{A S L}}{ }^{T} \dot{\boldsymbol{\Delta}}=\left(\left[\begin{array}{cccc}
k_{1} & 0 & \ldots & 0 \\
0 & k_{2} & \ldots & 0 \\
: & : & : & : \\
0 & 0 & \ldots & k_{5}
\end{array}\right] \boldsymbol{\Delta}\right)^{T} \dot{\boldsymbol{\Delta}}
$$

The elements of $\boldsymbol{\Delta}$ and $\boldsymbol{F}_{\boldsymbol{A} \boldsymbol{S} \boldsymbol{L}}$ in (9) are the elongation and the force of the ASLs, respectively. $k_{i}$ in this equation represent the stiffness of the ASL of the corresponding joint. The ASL elongation, $\boldsymbol{\Delta}$, is calculated as:

$$
\boldsymbol{\Delta}=2\left(h+\frac{t_{A S L}}{2}\right) \sin \frac{\boldsymbol{\theta}}{2}
$$

Replacing the energy storage rate of the ASLs in (2) yields (11) which correlates the tension in the tendon with the joint angles and their stiffness:

$$
\boldsymbol{F}_{\boldsymbol{t}}^{T} \boldsymbol{T}=\boldsymbol{f}^{T} \boldsymbol{J}+\left(h+\frac{t_{A S L}}{2}\right)^{2}\left(\boldsymbol{K}_{\boldsymbol{A S L}} \sin \boldsymbol{\theta}\right)^{T}
$$

We use this model to study the effect of joint stiffness on the reconfiguration of the finger and the compliance of the end-effector. The activation of different joints changes the free motion trajectory of the end-effector. The compliance of the finger for each trajectory can be controlled through the joints stiffness. Joints 1 and 8 of the robogami finger, with their opposite direction of actuation, influence the compliance of the finger significantly. Here, we study the effect of activation of these joints. This study signifies the importance of the model and the simulation results in understanding the potentials of different designs for under-actuated robogamis. For each simulation, we report the trajectory of the end-effector and the final 

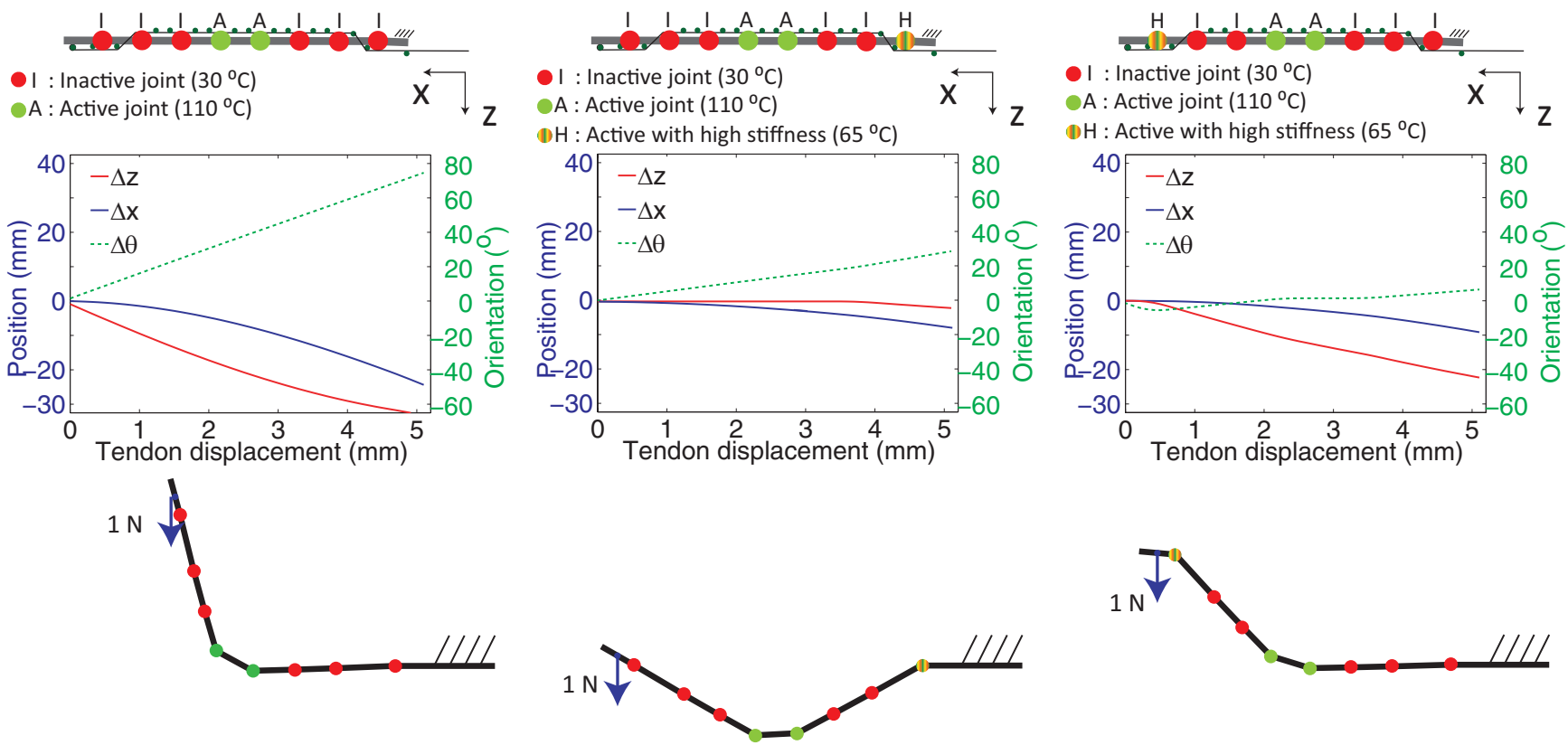

(a)

(b)

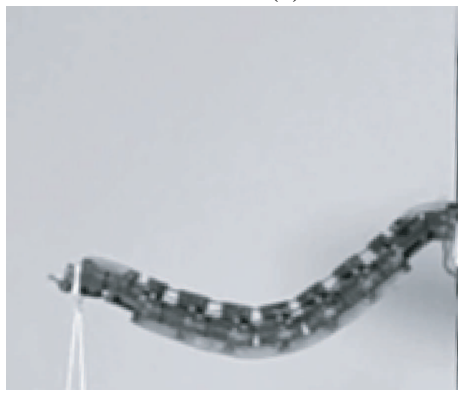

(e) (c)

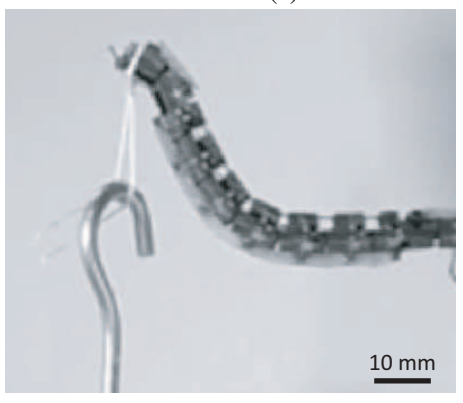

(f)

Fig. 4: The displacement of the end-effector with three different stiffness settings under $1 N$ load in $\mathrm{z}$ direction. In the first case, joints 4 and 5 are at high temperature $\left(110^{\circ} \mathrm{C}\right)$ and all the other joints are at room temperature (a). In the second case, the first joint is activated at a lower temperature $\left(65^{\circ} \mathrm{C}\right)$. Given the higher stiffness of the joint and its smaller actuation moment, the free displacement of the finger is similar to the previous case. The trajectory of the end-effector under load is, however, significantly different (b). In the third case, joint 8 is activated at $65^{\circ} C$. The activation of this joint also does not change the free displacement considerably but the rotational compliance of the end-effector under load is remarkably increased compared to the first case (c). The final configuration of the finger in experiments are presented for comparison (d-f). The overall behavior follows the one predicted in simulation. The main reason for the discrepancy is the neglected effect of the tendon friction with its channel.

configuration of the finger that was estimated by the model. To verify the simulation results we tested the finger with the same stiffness setting and contact load and compared the final configuration of the finger with the simulated configuration.

The motion of the robogami finger under load in $\mathrm{z}$ direction is simulated with three different stiffness settings, presented in Fig. $4 a-4 c$. The contact force increases with the motion of the end-effector in the $\mathrm{z}$ direction at the rate of $50 \mathrm{~N} / \mathrm{mm}$ and is saturated at $1 \mathrm{~N}$. The linear compliance of the end-effector in $\mathrm{z}$ direction and its rotational stiffness are dependent on the stiffness assigned to different joints. The first simulation, Fig. $4 \mathrm{a}$, is the reference to verify the effect of activation of the first and the last joints. In this case, joints four and five are active at their most compliant state at $110{ }^{\circ} \mathrm{C}$ with linear stiffness of $2.6 \mathrm{~N} / \mathrm{mm}$. The other joints in this case are inactive and at room temperature with linear stiffness of $110 \mathrm{~N} / \mathrm{mm}$ (for more detailed information regarding the characterization process and the stiffness variation of the ASL with temperature refer to [29]). For this setting, the motion of the finger is not effected by the load and it is characterized by high linear stiffness for the end-effector in $\mathrm{z}$ direction and high rotational stiffness. Activating the first joint makes the finger considerably more compliant in the $\mathrm{z}$ direction, Fig. 4b. In this case, the first joint is activated at $65{ }^{\circ} \mathrm{C}$ with the linear stiffness of $5.5 \mathrm{~N} / \mathrm{mm}$ and the setting for the other joints is similar to the previous case. The activation of the first joint at this temperature does not significantly affect the free displacement of the finger. However, the trajectory of the end-effector changes considerably under load and the position of the end-effector in $\mathrm{z}$ direction stays without much change during the actuation.

Note that actuating the first joint in the opposite direction is more effective for changing the compliance of the finger compared to having a passive joint. The increased tendon tension amplifies the moment applied to the first joint by the contact force and makes the finger more responsive, compliant, 
to the contact forces. In order to limit the deformation of the base joint in free displacement, to have similar free motion, the tendon is placed closer to the neutral plane for the first joint and this joint is activated with a lower temperature $\left(65^{\circ} \mathrm{C}\right)$, higher stiffness, compared to the rest of active joints. This design for the tendon distance and temperature setting for the first joint, results in similar free motion trajectories for the first two cases of Fig. 4. The compliance of the end-effector, however, is significantly different for these two cases which results in different trajectories and final configuration under load. If the same tendon distance was assigned to the first and other active joints in this case, higher stiffness for the first joint would have been required to achieve the same response. However, working at lower temperatures, for assigning higher stiffness, is not desired due to the sharp stiffness variation at temperatures lower than $60{ }^{\circ} \mathrm{C}$ [29].

The activation of the last joint also affects the compliance of the end-effector as presented in Fig. 4c. Given the small distance from this joint to the end-effector, the compliance in $\mathrm{z}$ direction does not change much in this case. The rotational compliance of the last link, however, changes considerably when this joint is activated. As was the case for the first joint, activation of the last joint at $65^{\circ} \mathrm{C}$ does not affect the trajectory of the free motion but under contact load the trajectory of the end-effector changes considerably and the orientation of the last link remains almost constant under load. By increasing the lateral and rotational compliance, the manipulator becomes self-adaptive in these directions to an assigned degree. This is desirable for safe interaction with sensitive environment. Compared to a fully actuated system, this approach is superior since it requires much less computation and control effort and only high level control on the joint stiffness and the overall behavior is required.

We tested the robogami finger with the conditions similar to the simulations to verify the results, Fig. 4d-4f. In the experiments the duty cycle for the heaters were set based on the readings from a thermal camera for reaching the desired temperature setting at each joint. The tendon displacement corresponding to the displacement used in simulation is applied using an electric motor, Dynamixel MX-28. The final configuration of the finger with different temperature settings are presented in Fig. 4 for comparison. The overall behavior is similar to the one predicted by simulation. The main reason for the discrepancy is the neglected tendon friction in the model. We considered constant tendon tension for all joints. Due to the friction, however, the tendon tension decreases from the base to the tip of the finger. So in simulation results we underestimated the motion of the joints closer to the base and overestimated the motion for the joints closer to the tip. Other sources of error such as difference between the ASLs of different joints and errors in the temperature settings also contribute to the discrepancy between simulation predictions and experiments.

We demonstrated the compliance control of the robogami finger in different directions. In the next Section, we present grasp modes corresponding to the same compliance settings which we have studied for a robogami finger in this Section.

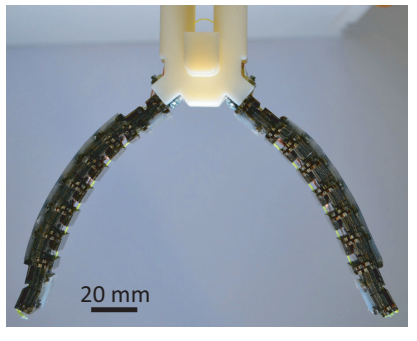

(a) (b)

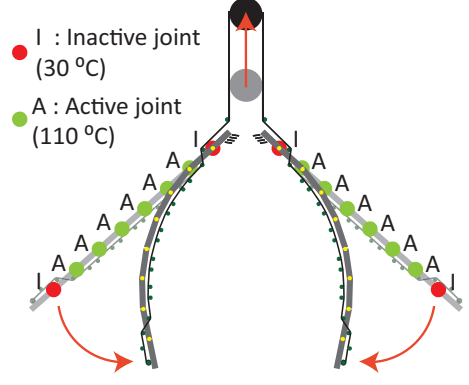

Fig. 5: The overview of the robogami gripper. The gripper consists of two fingers similar to the one of Fig. 3. A single input actuates both fingers through a pulley (a). The schematic of the gripper and its motion with the setting for power grasp (b). The green and red dots represent the active and inactive joints, respectively.

\section{UNDER-ACTUATED ROBOGAMI GRIPPER WITH ADJUSTABLE STIFFNESS JOINTS}

The tendon-driven robogamis provide a platform for designing inherently soft robots. The compliance in these robots originate from their redundant DoFs and under-actuation. Here we study the application of tendon-driven robogamis in a gripper. The conformity of such a gripper allows it to orient its contact points to apply forces in proper direction for a robust grasp. By implementing the adjustable stiffness joints such a gripper can perform different grasp modes based on the joints stiffness. The overall design of the gripper with two fingers is presented in Fig. 5. The actuation from the slider is divided between the two fingers through the tendon and the pulley. Depending on the stiffness setting for the joints, the tendon displacement drives different joints in the robogami. In the schematic of the gripper, Fig. 5b, the active and inactive, soft and stiff, joints are marked. In this Section, we demonstrate two different grasp modes: power grasp and precision grasp. For power grasp the object is enveloped by the fingers and is secured between the fingers and the palm of the gripper. In the precision grasp, the object is secured between the finger tips. These two modes of grasp require the activation of different joints. In each mode, the compliance of the fingers can also be adjusted by setting different levels of joints stiffness. In the present design, the activation of the first and the eighth joints increases the compliance of the actuation significantly (as discussed in Section III). In the last part of this Section, we compare the grasp of a soft object in two different modes for highlighting the effect of the activation of the first joint on the finger's compliance.

\section{A. Power grasp}

In the "power grasp" mode, the gripper secures the object between the fingers tips and the base. To perform the power grasp, joints 2-7 are activated at their most compliant state. The schematic of Fig. 5b presents the stiffness assignment and the actuation overview. The redundant DoFs of the fingers and the inherent softness of the under-actuated gripper, allows the last link of each finger to adapt its orientation to apply forces in the the proper direction for a robust grasp of the object as presented in Fig. 6. For larger objects, the overall compliance 


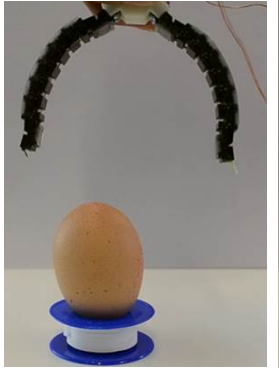

(a)

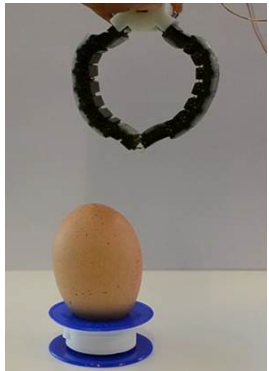

(b)

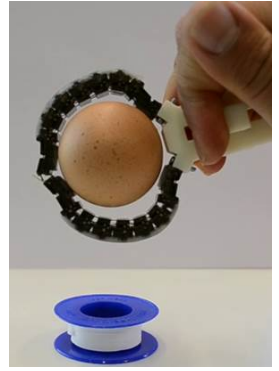

(c)
Fig. 6: The "power grasp" mode: all joints except for J1 and J8 are active: the initial state (a); the free motion (b); the grasp of an egg (c). The redundant DoF of the fingers in this mode of operation allows the last link to orient itself for applying the contact force at the proper direction for a stable grasp.

and conformity of the fingers allows them to take the shape of the object and envelope it. For the smaller object, like an egg, the self-adaptability of the last link illustrates the conformity of the gripper and the softness of the grasp.

\section{B. Precision grasp}

The second grasp mode of the gripper we study is the "precision grasp" of small objects between the finger tips. In this case, large displacement and small orientation change at the tip are required. So the base joints, 2 and 3, are activated in this mode as presented in the first schematic of Fig. 7d. The orientation of the end-effector for grasping small objects has to be adjusted based on the contact force and the size of the object. One approach is to reconfigure and lock the tip joint of

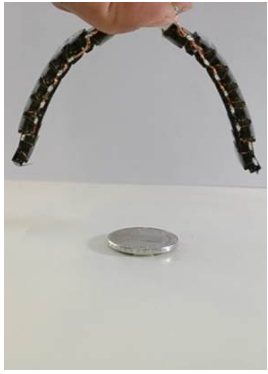

(a)

$\left(30^{\circ} \mathrm{C}\right)$
A : Active join $\left(110^{\circ} \mathrm{C}\right)$ $\mathrm{H}$ : Active with high
stiffness $\left(65^{\circ} \mathrm{C}\right)$

iffness $\left(65^{\circ} \mathrm{C}\right)$

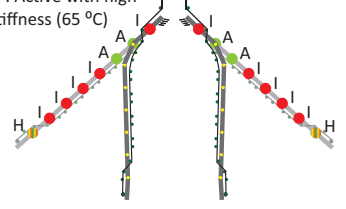

Precision grasp mode

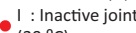

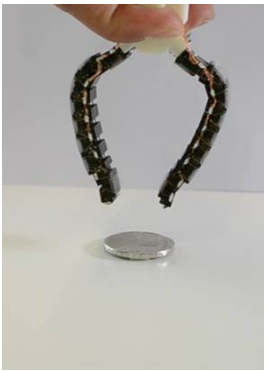

(b)

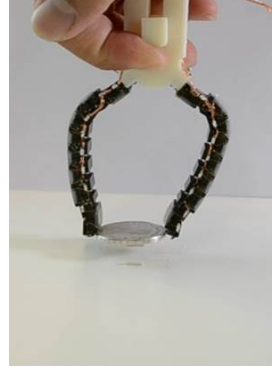

(c)

\section{Grasp compliance}

As mentioned in the previous Section, the compliance of the robogami fingers can be adjusted by changing the stiffness of the active joints and also by activating additional joints. In the present design, the activation of the first joint significantly

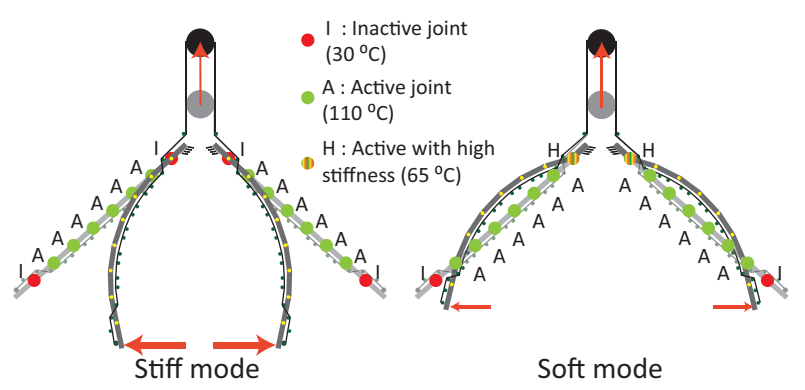

(a)

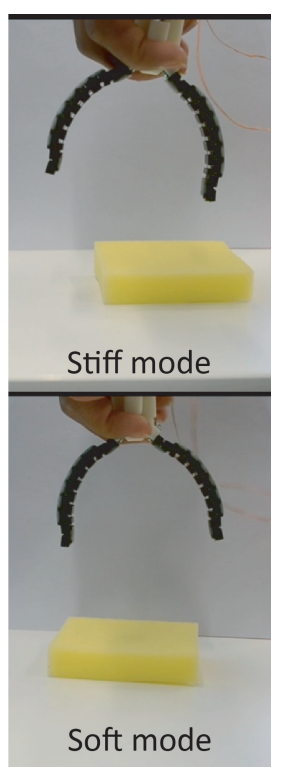

(b)

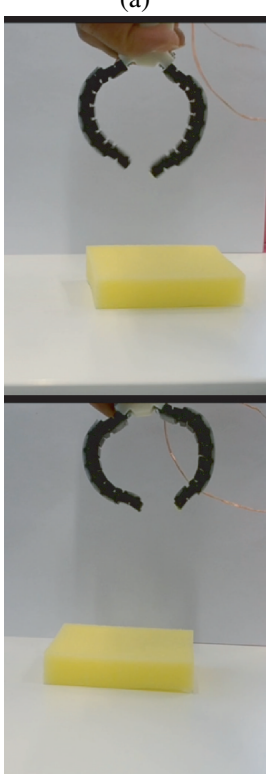

(c)

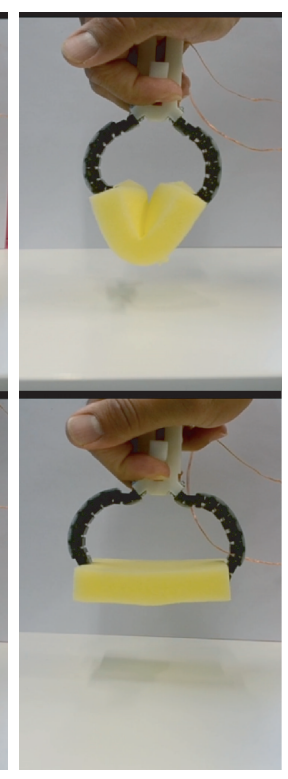

(d)
Fig. 8: The contact forces can be controlled by adjusting the compliance of the fingers. Activation of the first joint considerably increases the lateral compliance of the fingers which is desirable for handling soft and sensitive objects (a). In grasp with the soft setting, the force that the end-effector can apply is limited. To contrast the behavior of the gripper in soft and stiff modes, the grasp of a soft sponge is studied: the initial state (b); the free motion, Given the higher stiffness of the base joint and its lower actuation moment, the free motion of the gripper in similar in both settings (c); the grasp of the sponge, with the stiff setting, the gripper can apply large enough forces to deform the foam but with the soft setting the contact force is limited and the end-effector does not deform the foam (d).
Fig. 7: The "precision grasp" mode. The less number of DoF are active which results in less compliant and more controlled motion of the end-effector. The initial state (a); the free motion (b); the grasp of a $5 \mathrm{chf}$ coin, $31.5 \mathrm{~mm}$ in diameter and $13.2 \mathrm{gr}$ in mass (c). To facilitate the precision grasp, the eighth joint is also activated at $65^{\circ} \mathrm{C}$. This increases the rotational compliance of the end-effector which improves the contact point orientation (d). the finger in the proper orientation based on the object. Here temperature $\left(65^{\circ} \mathrm{C}\right)$ to increase the rotational compliance of the end-effector. The schematics of Fig. $7 \mathrm{~d}$ present how the activation of the last joint helps with the self adaptability of displacement and the grasping of a $5 \mathrm{CHF}$ coin $(t: 2.4 \mathrm{~mm}$ $\phi: 31.5 \mathrm{~mm}, \mathrm{~m}: 13.2 \mathrm{gr}$ ) in this mode of operation. We should point out that the stiffness setting for the tip is a delicate task since excessive compliance of the end-effector can diminish the grasp stability. 
increases the compliance of the finger. The increased compliance of the finger is desired in grasping soft and delicate objects since the gripper does not apply large contact forces in this mode. The controllable compliance of the fingers allows us to limit the contact forces at a desired magnitude without requiring any feedback. To illustrate this, we compare the grasping of a foam with two different compliance settings. In the stiff mode, joints 2-7 are active. For the soft mode, we activate the same joints, 2-7, and to increase the compliance, we also activate the first joint but at higher stiffness, $65^{\circ} \mathrm{C}$. The schematics of Fig. 8a illustrates the temperature setting and the motion of the gripper with these two compliance settings. The free motion of the gripper in these two modes are similar as presented in Fig. 8c. But when the gripper comes in contact with an object, the increased tendon tension along with the moment applied by the contact forces moves the first joint back when its activated. This results in limited contact force and hence inherently soft performance as presented in Fig. 8d. In the grasp with stiff setting, the end-effector applies large enough forces to deform the foam. However, with the soft grasp setting, the contact force is limited and applying more input displacement causes the contact point to come closer to the base but their distance and hence the applied force to the foam does not change considerably.

In this Section, we demonstrated two modes of grasp of a robogami gripper, power and precision grasps. For each mode, the compliance of the fingers and hence the contact forces can be controlled by adjusting the stiffness of the active joints or activating additional DoFs. We demonstrated this by grasping a soft foam with two different compliance settings.

\section{CONClusion}

Novel actuation technologies in the field of soft robotics offer self adaptability, safe interaction, low cost, and customizability which make them a desirable choice for robotic hands and grippers. A versatile robotic hand requires different configurations, actuation patterns, and compliance settings for performing different tasks. In this paper, we presented a tendon-driven robogami gripper with adjustable stiffness joints. Through adjusting the joints stiffness, we can assign the initial configuration of the gripper, its actuation pattern, and the compliance of the grasp.

The layer-by-layer manufacturing of robogamis along with the precise quasi-2D fabrication methods enable us to design and fabricate fingers with many DoF in confined space. Given the multiple and redundant DoFs, robogami fingers can conform to the shape of the objects in contact. The adjustable stiffness joints of the robogami fingers enable us to control the operating mode of the gripper. Based on the significant variation in the elastic properties and the compatibility with the layer-by-layer manufacturing of the robogamis, we used SMP for controlling the joints stiffness. The proposed design for the robogami finger can be easily scaled up or down and the sequence of the DoFs can be customized without any significant effort in design and manufacturing.

To study different finger designs for the gripper and the activation pattern of the joints, we modeled the behavior of the under-actuated Robotogami fingers in free motion and under contact load. This model allows us to simulate the motion of the finger with different stiffness settings. We used the results of such simulations in designing a sequence of DoFs that allow significant compliance change by activation of different joints. In this paper we focused on motion in plane and demonstrated this compliance control in simulation and verified the result in experiments for the final design of the finger.

We used a gripper with two robogami fingers to demonstrated different modes of operation based on the stiffness setting. We first demonstrated the self adaptability of the robogami gripper by activating six joints in each finger. Upon actuation, the the fingers conform to the shape of the object for applying forces at the proper direction for a stable grasp. We demonstrated the robust grasp of an egg in this mode, the power grasp. Next, we presented the precision grasp of a coin by activating only two joints at the base which results in a stiffer mode of operation and more controlled motion of the end-effector. In this mode of operation, the last joint which is actuated in the opposite direction is also activated, but at higher stiffness, for allowing the last link to better adjust its orientation for a robust grasp. Finally, we studied the compliance of the grasp at two different stiffness settings by comparing the deformation of a foam. We demonstrated limited contact force in the soft mode of operation which allows safe interaction in sensitive environment without the need for any complex sensors or controller.

In this research, we focused on robogamis with motion in plane and demonstrated compliance control. The same concept can be generalized for 3D motions and controlling the compliance along other directions, which can be used in more capable robotic hands. The model that is presented in this work can easily be adjusted to study the motion of more complicated structures to find optimum design and to assign joints stiffness for performing different tasks.

\section{ACKNOWLEDGMENT}

This work was supported by Swiss National Center for Competence in Research (NCCR) in Robotics.

\section{REFERENCES}

[1] N. Doshi, B. Goldberg, R. Sahai, N. Jafferis, D. Aukes, R. J. Wood, and J. A. Paulson, "Model driven design for flexure-based microrobots," in Proc. IEEE/RSJ Int. Conf. Int. Robot. and Sys., Sept 2015, pp. 41194126.

[2] R. J. Wood, S. Avadhanula, R. Sahai, E. Steltz, and R. S. Fearing, "Microrobot design using fiber reinforced composites," J. Mech. Design, vol. 130, no. 5, pp. 052 304-052 304, 2008, 10.1115/1.2885509.

[3] A. Firouzeh, Y. Sun, H. Lee, and J. Paik, "Sensor and actuator integrated low-profile robotic origami," in Proc. IEEE/RSJ Int. Conf. Int. Robot. and Sys., Nov 2013, pp. 4937-4944.

[4] L. Hines, V. Arabagi, and M. Sitti, "Shape memory polymer-based flexure stiffness control in a miniature flapping-wing robot," IEEE Tran. Robot., vol. 28, no. 4, pp. 987-990, 2012.

[5] S. Hirose and Y. Umetani, "The development of soft gripper for the versatile robot hand," Mech. Mach. Theory, vol. 13, no. 3, pp. $351-$ 359, 1978.

[6] W. Ying, C. Yonghua, R. Tao, C. Qiao, Y. Changxin, Y. Yang, and L. Yingtian, "A novel, variable stiffness robotic gripper based on integrated soft actuating and particle jamming," Soft Robotics, vol. 3(3), pp. 134-143, Sep. 2016. 
[7] D. M. Aukes, B. Heyneman, J. Ulmen, H. Stuart, M. R. Cutkosky, S. Kim, P. Garcia, and A. Edsinger, "Design and testing of a selectively compliant underactuated hand," The Int. J. Robot. Res., 2014.

[8] L. U. Odhner, L. P. Jentoft, M. R. Claffee, N. Corson, Y. Tenzer, R. R. Ma, M. Buehler, R. Kohout, R. D. Howe, and A. M. Dollar, "A compliant, underactuated hand for robust manipulation," The Int. J. Robot. Res., 2014.

[9] J. Hughes, U. Culha, F. Giardina, F. Guenther, A. Rosendo, and F. Iida "Soft manipulators and grippers: A review," Frontiers in Robotics and $A I$, vol. 3, p. 69, 2016.

[10] A. Degani, H. Choset, A. Wolf, and M. A. Zenati, "Highly articulated robotic probe for minimally invasive surgery," in Proc. IEEE Int. Conf. Robot. and Autom., pp. 4167-4172.

[11] J. Choi, S. Hong, W. Lee, S. Kang, and M. Kim, "A robot joint with variable stiffness using leaf springs," IEEE Tran. Robot., vol. 27, no. 2, pp. 229-238, April 2011.

[12] S. Wolf, G. Grioli, O. Eiberger, W. Friedl, M. Grebenstein, H. Hppner, E. Burdet, D. G. Caldwell, R. Carloni, M. G. Catalano, D. Lefeber, S. Stramigioli, N. Tsagarakis, M. V. Damme, R. V. Ham, B. Vanderborght, L. C. Visser, A. Bicchi, and A. Albu-Schffer, "Variable stiffness actuators: Review on design and components," IEEE/ASME Trans. Mech., vol. 21, no. 5, pp. 2418-2430, Oct 2016.

[13] R. A. Stavenuiter, L. Birglen, and J. L. Herder, "A planar underactuated grasper with adjustable compliance," Mech. and Mach. Theory, 2016.

[14] M. E. Giannaccini, I. Georgilas, I. Horsfield, B. H. P. M. Peiris, A. Lenz, A. G. Pipe, and S. Dogramadzi, "A variable compliance, soft gripper," Auto. Robots, vol. 36, no. 1, pp. 93-107, 2014.

[15] K. C. Galloway, K. P. Becker, B. Phillips, J. Kirby, S. Licht, D. Tchernov, R. J. Wood, and D. F. Gruber, "Soft robotic grippers for biological sampling on deep reefs," Soft Robotics, vol. 3, no. 1, pp. 23-33, 2016.

[16] J. Ou, L. Yao, D. Tauber, J. Steimle, R. Niiyama, and H. Ishii, "jamsheets: Thin interfaces with tunable stiffness enabled by layer jamming," in Proceedings of the 8th International Conference on Tangible, Embedded and Embodied Interaction, ser. TEI '14. New York, NY, USA: ACM, 2013, pp. 65-72. [Online]. Available: http://doi.acm.org/10.1145/2540930.2540971

[17] S. Diller, C. Majidi, and S. H. Collins, "A lightweight, low-power electroadhesive clutch and spring for exoskeleton actuation," in 2016 IEEE International Conference on Robotics and Automation (ICRA), May 2016, pp. 682-689.

[18] A. Firouzeh, S. S. Mirrazavi Salehian, A. Billard, and J. Paik, "An under actuated robotic arm with adjustable stiffness shape memory polymer joints," in Proc. IEEE Int. Conf. Robot. and Autom., May 2015, pp. 2536-2543.

[19] M. A. McEvoy and N. Correll, "Thermoplastic variable stiffness composites with embedded, networked sensing, actuation, and control," $J$. Comp. Mat., 2014.

[20] W. Shan, T. Lu, and C. Majidi, "Soft-matter composites with electrically tunable elastic rigidity," Smart Mater. Struct., vol. 22, no. 8, p. 085005 , 2013.

[21] A. Firouzeh, M. Salerno, and J. Paik, "Soft pneumatic actuator with adjustable stiffness layers for multi-dof actuation," in Proc. IEEE/RSJ Int. Conf. Int. Robot. and Sys., Sept 2015, pp. 1117-1124.

[22] T. Ranzani, M. Cianchetti, G. Gerboni, I. D. Falco, and A. Menciassi, "A soft modular manipulator for minimally invasive surgery: Design and characterization of a single module," IEEE Tran. Robot., vol. 32, no. 1, pp. 187-200, Feb 2016

[23] A. A. Stanley and A. M. Okamura, "Controllable surface haptics via particle jamming and pneumatics," IEEE Trans. Haptics, vol. 8, no. 1, pp. 20-30, Jan 2015.

[24] N. G. Cheng, A. Gopinath, L. Wang, K. Iagnemma, and A. E. Hosoi, "Thermally tunable, self-healing composites for soft robotic applications," Macromol. Mater. Eng., pp. n/a-n/a, 2014.

[25] W. Wang, H. Rodrigue, and S.-H. Ahn, "Deployable soft composite structures," Sci. Rep., vol. 6, p. 20869, 2016.

[26] J. Shintake, B. Schubert, S. Rosset, H. Shea, and D. Floreano, "Variable stiffness actuator for soft robotics using dielectric elastomer and lowmelting-point alloy," in Proc. IEEE/RSJ Int. Conf. Int. Robot. and Sys., Sept 2015, pp. 1097-1102.

[27] J. Viau, P. Chouinard, J. P. L. Bigue, G. Julio, F. Michaud, and J. S. Plante, "Tendon-driven manipulator actuated by magneto-rheological clutches exhibiting both high-power and soft motion capabilities," IEEE/ASME Trans. Mech., vol. PP, no. 99, pp. 1-1, 2016.

[28] T. Takeda, Y. Shindo, and F. Narita, "Flexural stiffness variations of woven carbon fiber composite/shape memory polymer hybrid layered beams," J. Comp. Mat., vol. 49, no. 2, pp. 209-216, 2015.
[29] A. Firouzeh, M. Salerno, and J. Paik, "Stiffness control with shape memory polymer in underactuated robotic origamis," IEEE Tran. Robot., vol. PP, no. 99, pp. 1-13, 2017.

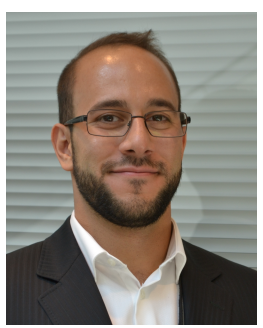

Amir Firouzeh ( $\mathrm{M}^{\prime}$ 17) received the B.Sc. and M.Sc. degrees in mechanical engineering from Tehran and Sharif Universities, Tehran, Iran, in 2009 and 2012, respectively and the Ph.D. degree in Robotics, Control and Intelligent Systems from Swiss Federal Institute of Technology, Lausanne, Switzerland, in 2017. He is currently a Research Assistant with the Reconfigurable Robotics Laboratory, Swiss Federal Institute of Technology, Lausanne, Switzerland. His research interests include novel sensing, actuation, and manufacturing technologies for soft robots towards their application in inherently compliant wearable devices for safe human interaction.

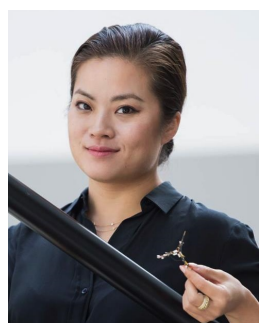

Jamie Paik (M' 07) is director and founder of Reconfigurable Robotics Lab (RRL) of Swiss Federal Institute of Technology (EPFL), Lausanne, Switzerland, and a core member of Swiss NCCR robotics group. RRLs research leverages expertise in multimaterial fabrication and smart material actuation. She received her PhD in Seoul National University on designing humanoid arm and a hand. During her Postdoctoral positions in ISIR (Institut des Systems Intelligents et de Robotic) in Universitat Pierre Marie Curie, Paris VI, she developed laparoscopic tools that are internationally patented and commercialized. At Harvard Universitys Microrobotics Laboratory, she started developing unconventional robots that push the physical limits of material and mechanisms. Her latest research effort is in soft robotics and self-morphing Robogami (robotic orgami) that transforms its planar shape to $2 \mathrm{D}$ or $3 \mathrm{D}$ by folding in predefined patterns and sequences, just like the paper art, origami. 